\title{
THE TRIANGULATION OF TOE-IN STYLE STEREO CAMERA
}

\author{
Kosuke Katada ${ }^{a}$, Shenjing Chen ${ }^{\text {a }}$, Lifeng Zhang ${ }^{\mathrm{a}}$ \\ aKyusyu Institute of Technology, 1-1 Sensui-cho Tobata-ku Kitakyuusyuu-shi Fukuoka-ken, 804-8550, Japan \\ o349408k@mail.kyutech.jp
}

\begin{abstract}
This study aimed to propose a triangulation by using a consumer $3 \mathrm{D}$ camera in order to measure a distance. In traditional method, error of distance measurement was large. The consumer 3D camera is different from a dedicated measurement stereo camera; the optical axes is installed so as to cross in front of lens several meters away (toe-in style), in order to give a sense of reality of human being's visual characteristic. We examine accuracy of proposed method by mesh data construction.
\end{abstract}

Keywords: Triangulation, Distance measurement, 3D camera, Mesh data construction

\section{Introduction}

Stereo camera that has two lenses is used in various fields. In the image field, Stereo camera is used for 3D movies and $3 \mathrm{D}$ video games. In the industrial field, it is used as a device for measuring size, shape, and distance from the captured image. Parallel stereo camera is used if you want a distance measurement. Parallel stereo camera enables fast distance calculation by using the principle of a simple triangulation because optical axes of the two lenses are installed in parallel. However, it is difficult for make completely parallel optical axes. Thus, the high accuracy device is expensive.

By contrast, the consumer 3D camera is not suitable for distance measurement because the optical axes is installed so as to cross in front of lens. Crossed optical axis complicates the distance calculation, it has adverse effect on the calculation accuracy.

Thus, it is necessary to use both the camera according to the situation. Being able to unify the camera, it is possible to construct the $3 \mathrm{D}$ data of the object while taking a 3D image. The consumer 3D camera (FinePix REAL 3DW3) was used in this experiment. The features of these consumer 3D camera has high portability and sold in low price.

\section{Stereo matching}

It is necessary to know the parallax of the two images is to use a triangulation. We were using a template matching as the method this time. I explain how to obtain the parallax by the method. First, template image is created by around point $\mathrm{P}$. Next, detect the corresponding point $\mathrm{Q}$ by band-like image. The difference of the coordinates of $\mathrm{P}$ and $\mathrm{Q}$ is parallax. In this study, Zero-mean Normalized Cross-Correlation (ZNCC) algorithm is utilized.

\section{Triangulation}

\subsection{Triangulation}

Common triangulation is a distance measuring method using the geometry. The distance to the object from the camera can be calculated by knowing the angle to the target from both ends of the baseline and baseline distance is known. In the triangle in Fig. 2, the distance $d$ can be

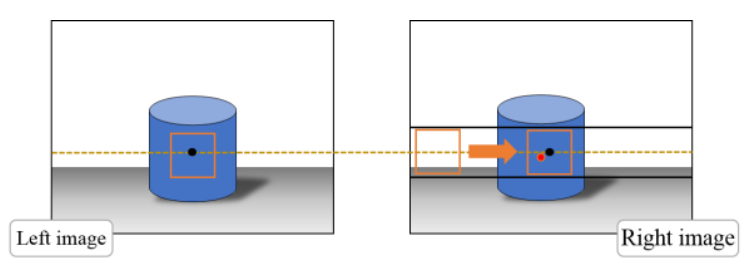

Fig. 1. Template matching 
calculated using the angle $\alpha, \beta$ and baseline $B$.

$\mathrm{d}=\mathrm{B} /\left(\frac{1}{\tan \alpha+\tan \beta}\right)$

\subsection{Traditional Triangulation}

Parallel stereo camera was used to measure distance. Distance formula was a parallel stereo camera only. In Fig. 3 , the distance $Z$ can be calculated using the focal length $f$, baseline $B$ and the parallax $x_{1}, x_{2}$ because triangle that is configured by two lenses and the object and triangle that is configured by two lenses and the projection plane are similar.

$$
\mathrm{Z}=\frac{B f}{\left(x_{1}+x_{2}\right)}
$$

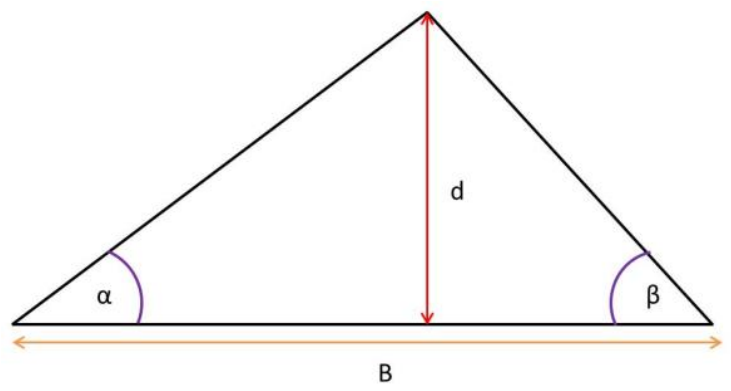

Fig. 2. Triangulation

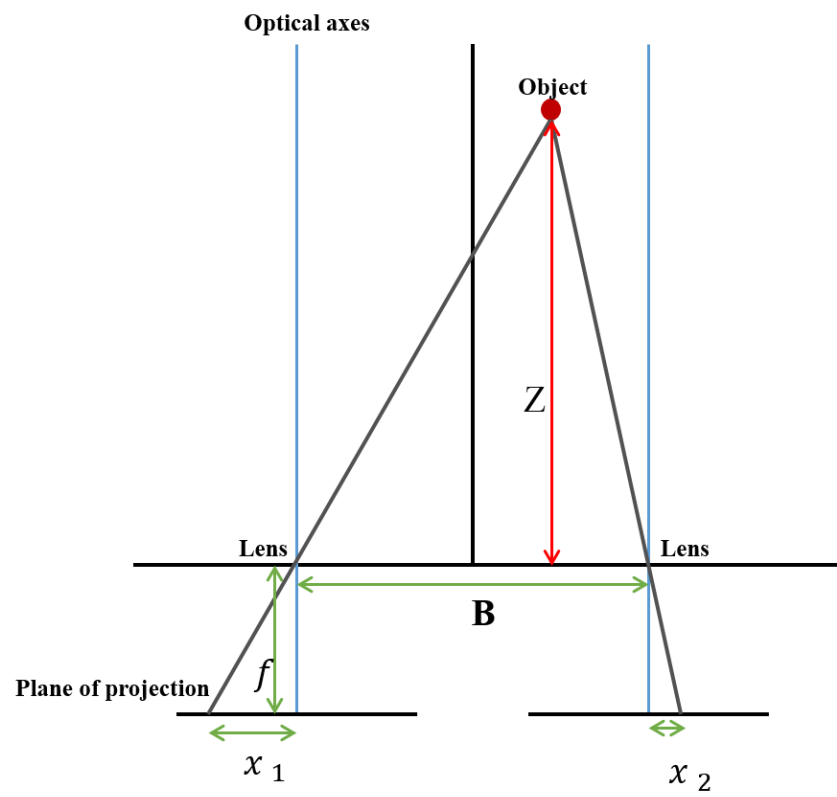

Fig. 3. Traditional Triangulation

\subsection{Proposed Triangulation}

The optical axis intersects the camera to be used this time. An error occurs when using the conventional equation. So, I propose a triangulation of the case where the optical axis intersects. It is possible to use (1) equation to be able to obtain the angle $\alpha$, and $\beta$. First, we find the angle $\theta$ the baseline and the optical axis of the left lens in Fig. 4 forms. The use of the distance $\mathrm{L}$ to the cross-point, the distance $\mathrm{B}$ between the cameras, it is possible to determine the angle $\theta$ According to the following equation.

$$
\theta=\tan ^{-1}\left(\frac{2 L}{B}\right)
$$

We derive $\emptyset_{1}$. It is possible $\emptyset_{2}$ obtained by the following equation using the parallax $\mathrm{x} 1$ distance $\mathrm{B}$ between the lenses, the focal length $f$ of the camera, projected image of the left camera.

$$
\emptyset_{1}=\tan ^{-1}\left(\frac{x_{1}}{f}\right)
$$

Further, $\emptyset_{2}$ can be obtained by the following formula it is possible to know the $\mathrm{x} 2$ of the right lens.

$$
\emptyset_{2}=\tan ^{-1}\left(\frac{x_{2}}{f}\right)
$$

Thus, $\alpha$ and $\beta$ are obtained by the following equation.

$$
\begin{aligned}
& \alpha=\theta-\emptyset_{1} \\
& \beta=\theta-\emptyset_{2}
\end{aligned}
$$

\section{Comparison}

We test the accuracy of proposed method by experiment. We measured up to $2000 \mathrm{~mm}$ from $500 \mathrm{~mm}$ by installing an object in front of the camera. Table 1 shows the results of the traditional method. Table 2 shows the results of the proposed method. 


\section{Simulation}

In order to make confirmation about the accuracy of the proposed method, several 3D mesh data creation examinations were performed. The test subjects are the faces of human being. This face has no obvious texture patterns on their surface. Figure 5(a) show the measurement targets and the rectangular frame shows the measuring range. Figure 5(b) show the 3D mesh data in the measuring range. Simulation results show that the shape of the $3 \mathrm{D}$ mesh data is created correctly. As described above, examined subjects have no obvious texture patterns on their

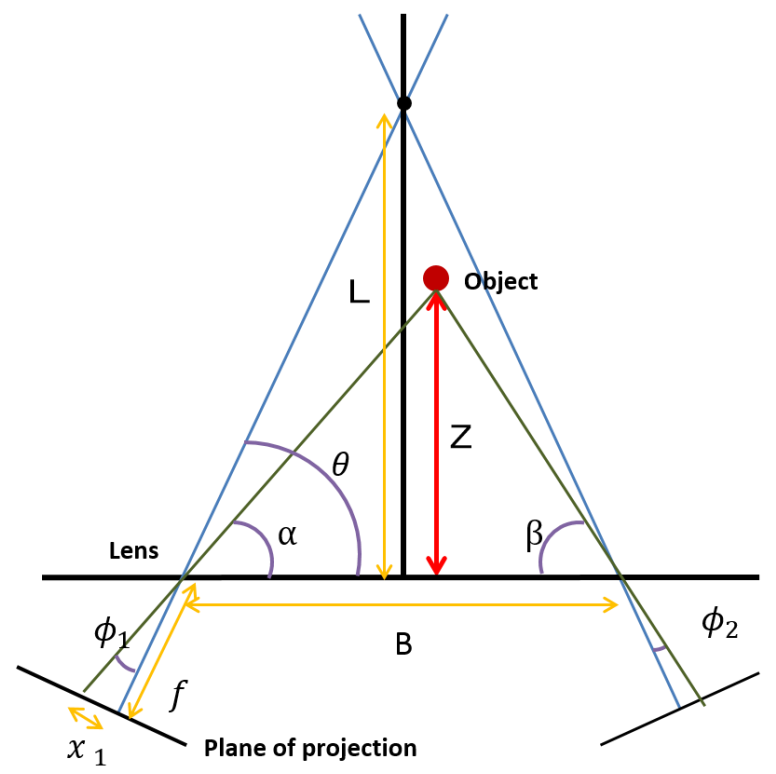

Fig. 4. Proposed Triangulation

Table 1. The result of the traditional method.

\begin{tabular}{|c|c|c|}
\hline $\begin{array}{c}\text { Real } \\
\text { Distance[mm] }\end{array}$ & $\begin{array}{c}\text { Measurement } \\
\text { distance[mm] }\end{array}$ & $\begin{array}{c}\text { Error of } \\
\text { measurement[mm] }\end{array}$ \\
\hline 500 & 614.37 & 114.37 \\
\hline 1000 & 1793.86 & 793.86 \\
\hline 1500 & 5567.95 & 4067.95 \\
\hline 2000 & -52275.22 & -54275.22 \\
\hline
\end{tabular}

Table 2. The result of the proposed method.

\begin{tabular}{|c|c|c|}
\hline $\begin{array}{c}\text { Real } \\
\text { distance[mm] }\end{array}$ & $\begin{array}{c}\text { Measurement } \\
\text { distance[mm] }\end{array}$ & $\begin{array}{c}\text { Error of } \\
\text { measurement[mm] }\end{array}$ \\
\hline 500 & 452.92 & -47.08 \\
\hline 1000 & 921.18 & -78.82 \\
\hline 1500 & 1416.12 & -83.88 \\
\hline 2000 & 1971.93 & -28.07 \\
\hline
\end{tabular}

surface; especially the skin and the hair largely influence the accuracy of corresponding point detection. Therefore, the surface of the created 3D mesh is not as smooth as the real subjects.

\section{Conclusion}

In this study, we first introduced the template matching algorithm for corresponding point detection. Then we proposed a new approach for adapting new triangulation method to a toe-in type stereo camera system. After the accuracy examination, we performed a practical simulation for the $3 \mathrm{D}$ mesh data. As a result, the proposed method have greater accuracy of long distance measurement than traditional method. In the simulation, we have succeeded in constructing roughly the $3 \mathrm{D}$ data of the object. However, distance measurement result on rugged surface or outline has low accuracy. It is caused by not finding corresponding point. Further consideration will be needed to yield any findings about the detection of the corresponding point on the rugged surface or outline.

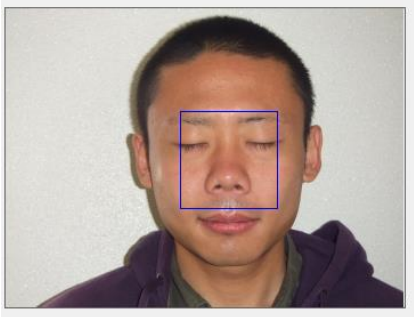

(a) Left image of face

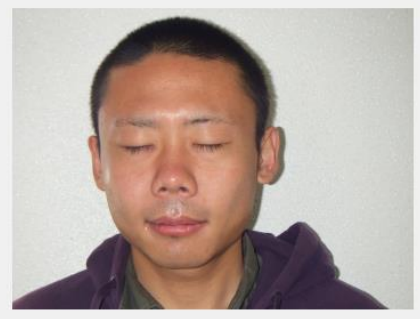

(b) Right image of face
Fig. 5. Face images

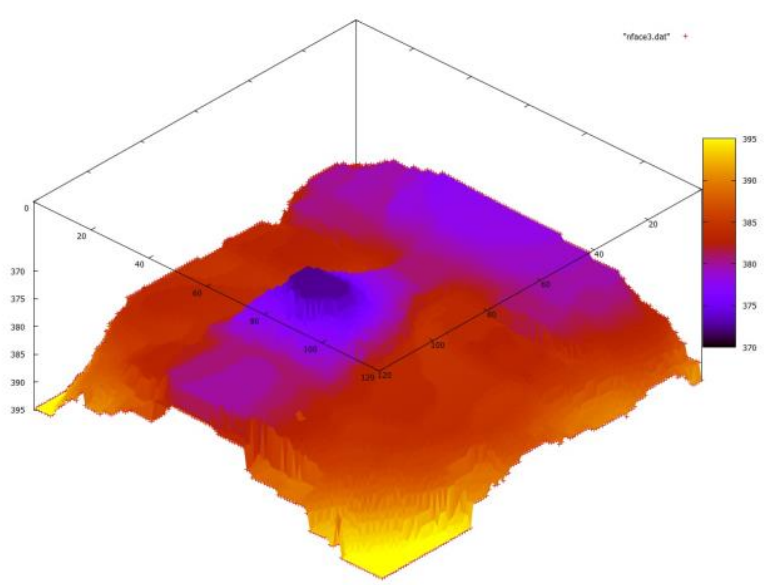

Fig. 6. 3D mesh data of face 


\section{References}

(1) M. Shimizu and M. Okutomi, Sub-pixel estimation error cancellation on area-based matching, International Journal of Computer Vision, vol.63, no.3, pp.207-224, 2005.

(2) D. Scharatein and R. Szeliski, A taxonomy and evaluation of dense two-frame stereo correspondence algorithms, International Journal of Computer Vision, vol.47, no.1-3, pp.7-42, 2002.

(3) T. Shibahara, T. Aoki, N. Nakajima and K. Kobayashi, A high-accuracy stereo correspondence technique using 1D band-limited phase-only correlation, IEICE Electronics Express, vol.5, no.4, pp.125-130, 2008.

(4) K. Takita, T. Aoki, Y. Sasaki, T. HIiguchi and K. Kobayashi, High-accuracy subpixel image registration based on phase-only correlation, IEICE Trans. Fundamentals, vol.E86-A, no.8, pp.1925-1934, 2003.

(5) K. Takita, M. A. Muquit, T. Aoki and T. Higuchi, A sub-pixel correspondence search technique for computer vision applications, IEICE Trans. Fundamentals, vol.E87-A, no.8, pp.1913-1923, 2004.

(6) A.-L. Hou, X. Cui, Y. Geng, W.-J. Yuan and J. Hou, Measurement of safe driving distance based on stereo vision, Proc. of the 6th International Conference on Image and Graphics, pp.902-907, 2011.

(7) H.-Y. Lin, P.-K. Huang, T.-Y. Lin, T.-T. Chang, C.-H. $\mathrm{Wu}$, C.-C. Hsiao and C.-K. Liao, Stereo matching architecture for 3D pose/gesture recognition and distance-measuring application, Proc. of International Conference on 3D Imaging, pp.1-6, 2013. 